\title{
The top quark pair production at ILC in the minimal gauge extension of the SM
}

\section{Vlasenko* and V. Kuksa}

Southern Federal University, Russia

E-mail: vlasenko91@list.ru, kuksa@list.ru

The process $e^{+} e^{-} \rightarrow t^{*} t^{*} \rightarrow b \bar{b} W^{+} W^{-}$is considered within the frame-work of the model of unstable particles with smeared mass. Consideration was fulfilled in the frame-work of the SM and the minimal gauge extension of the SM. We carry out the renormalization group analysis of coupling constants and make a comparison of the cross-section in both models.

The XXth International Workshop High Energy Physics and Quantum Field Theory September 24 - October 1, 2011

Sochi, Russia

\footnotetext{
${ }^{*}$ Speaker.

${ }^{\dagger}$ I would like to thank G.Vereshkov for his help in the considered model construction and analysis.
} 


\section{Introduction}

There are some motivations to consider extensions of the SM, which has some problems, such as problem of generations, special neutrino status, in particular neutrino mass, Higgs boson etc. Some of these problems can be solved in the gauge extensions of the SM. In this work, we consider the minimal gauge extension of the SM (MGE) which belongs to the five rank intermediate models of unification and is the simplest one. For neutrino mass and mixing description it is necessary to introduce right-hand neutrino, which interacts with additional gauge boson $Z^{\prime}$, therefore gauge group of the model is $U_{e m}(1) \times S U_{L}(2) \times S U_{C}(3) \times U_{R}(1)$. Higgs sector is modified by addition of new singlet scalar Higgs boson which interact with new $Z^{\prime}$ boson and right-hand neutrino, only.

To analyze any process of the model we have to connect new parameters with experimental restrictions. In our calculations we need to fix $Z^{\prime}$ boson parameters. We applied experimental restrictions from $L E P 2$ and $L H C$, and perform renormalization group (RG) analysis in the oneloop approximation.

Top quark physics is an important part of the future $e^{+} e^{-}$linear collider experiments [1]. In this work, we consider the process $e^{+} e^{-} \rightarrow t^{*} t^{\bar{*}} \rightarrow b \bar{b} W^{+} W^{-}$. The consideration is fulfilled in the frame-work of the model of unstable particles with a smeared mass (SMUP model) [2,3]. In our calculations, we account for NLO radiative EW and QCD factorizable corrections. We illustrate the role of the corrections and the difference between the standard and model total cross-section, angle differential cross-sections and invariant mass distribution.

\section{Minimal gauge extension of the SM}

Here, we consider gauge extension of the SM with extra neutral gauge boson, singlet Higgs boson and right-hand neutrino. Modern experimental restrictions on new coupling constant $g^{\prime}$ and mass of $Z^{\prime}$ are the following

$$
\frac{M_{Z^{\prime}}}{g^{\prime}} \gtrsim 7 \mathrm{TeV}(L E P 2) ; M_{Z^{\prime}} \gtrsim 1.8 \mathrm{TeV}(\mathrm{LHC})
$$

For RG analysis it is necessary to fix a reduction scheme, which is presented in Fig.1. The $\beta$ functions of MGE model are $\beta_{\text {em }}=4.1, \beta_{\text {weak }}=\frac{2}{3}, \beta_{\text {new }}=\frac{25}{6}$ and running couplings $\alpha_{k}\left(Q^{2}\right)$ give scale of unification which is presented in Fig.2.

$$
\alpha_{k}\left(Q^{2}\right)=\frac{\alpha_{k}\left(M_{Z}\right)}{1-\left(\beta_{k} / 2 \pi\right) \ln \left(Q^{2} / M_{Z}^{2}\right)} .
$$

Thus, taking into account the experimental restrictions and that scale of unification must be less than scale of grant unification, $R G$ analysis in one-loop approximation gives value of $Z^{\prime}$ mass more than $3.5 \mathrm{TeV}$.

\section{The model cross-section of the top-pair production and decay}

The process of top-pair production and decay $e^{+} e^{-} \rightarrow t^{*} t^{*} \rightarrow b \bar{b} W^{+} W^{-}$is presented in Fig.3. From this picture, one can see that full process contains $t \bar{t}$ unstable intermediate time-like state. In 


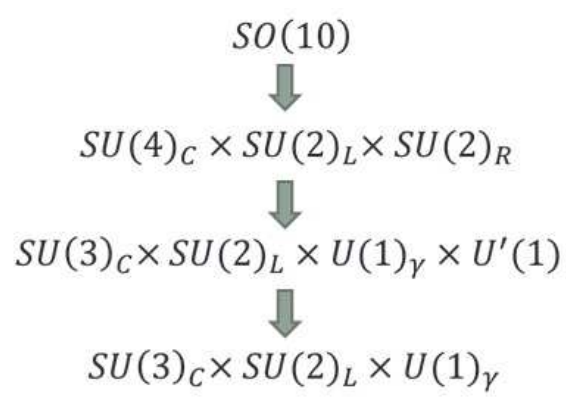

Figure 1: Reduction scheme.

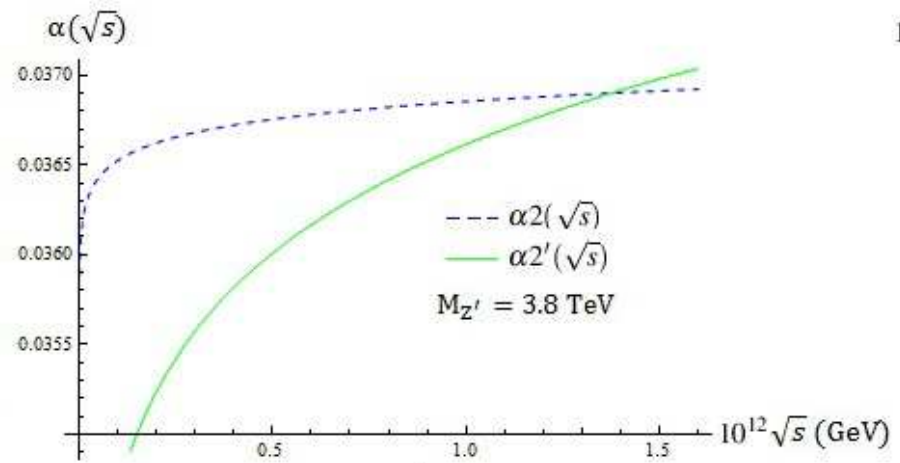

a)

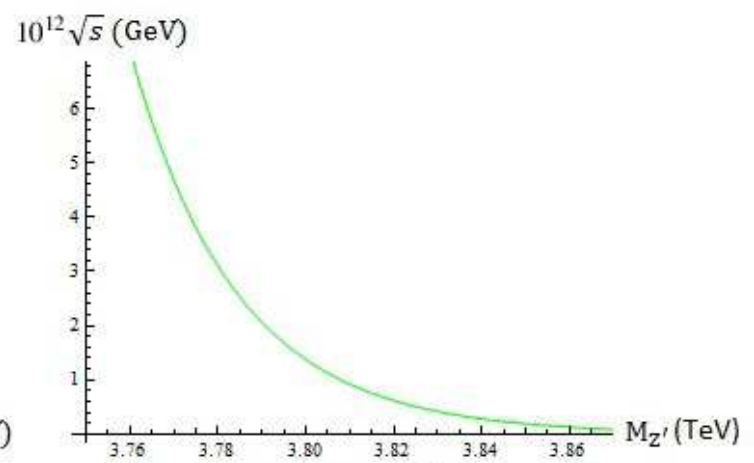

b)

Figure 2: a) Dependence of the coupling constants from $\sqrt{s}$. b) Scale dependence of unification from $M_{Z^{\prime}}$

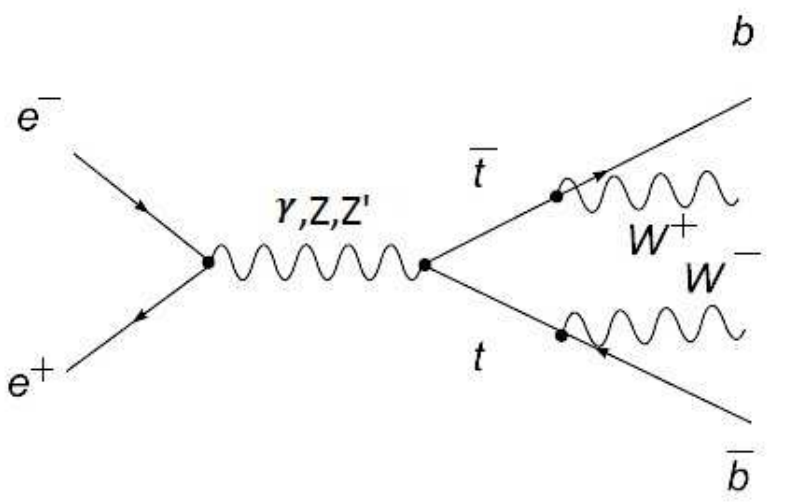

Figure 3: Feynman diagram for the process $e^{+} e^{-} \rightarrow b \bar{b} W^{+} W^{-}$.

the frame-work of the SMUP model [2] this process can be divided into two stages: $e^{+} e^{-} \rightarrow t^{*} \bar{t}^{*}$ and $t^{*} \bar{t}^{*} \rightarrow b \bar{b} W^{+} W^{-}$. Here, top-quarks should be treated as unstable particle with large widths.

Here, we derive the formula for cross-section of inclusive process which directly follows from the mass-smearing conception in the frame-work of the SMUP model. The model cross-section of 
the first reaction is [2]:

$$
\sigma\left(e^{+} e^{-} \rightarrow t^{*} \bar{t}^{*}\right)=\int_{m_{0}^{2}}^{s} \int_{m_{0}^{2}}^{\left(\sqrt{s}-m_{1}\right)^{2}} \sigma\left(e^{+} e^{-} \rightarrow t\left(m_{1}\right) \bar{t}\left(m_{2}\right)\right) \rho_{t}\left(m_{1}\right) \rho_{t}\left(m_{2}\right) d m_{1}^{2} d m_{2}^{2},
$$

where $m_{o} \approx 2 m_{b}$ is threshold value of the top mass, $\sigma\left(e^{+} e^{-} \rightarrow t\left(m_{1}\right) \bar{t}\left(m_{2}\right)\right)$ is the cross-section of tops production with random masses $m_{1}$ and $m_{2}, \rho_{t}(m)$ is probability density which describes mass smearing. In our calculations we take it in Lorentzian form [2]:

$$
\rho_{t}(m)=\frac{1}{\pi} \frac{m \Gamma_{t}(m)}{\left(m^{2}-M_{t}^{2}\right)^{2}+m^{2} \Gamma_{t}^{2}(m)},
$$

where $\Gamma_{t}(m)$ is total width of top quark with mass $m$. Because of $B r(t \rightarrow b W) \approx 1$, the expression (3.1) describes full process. The results of the model calculations are presented in Fig.4.

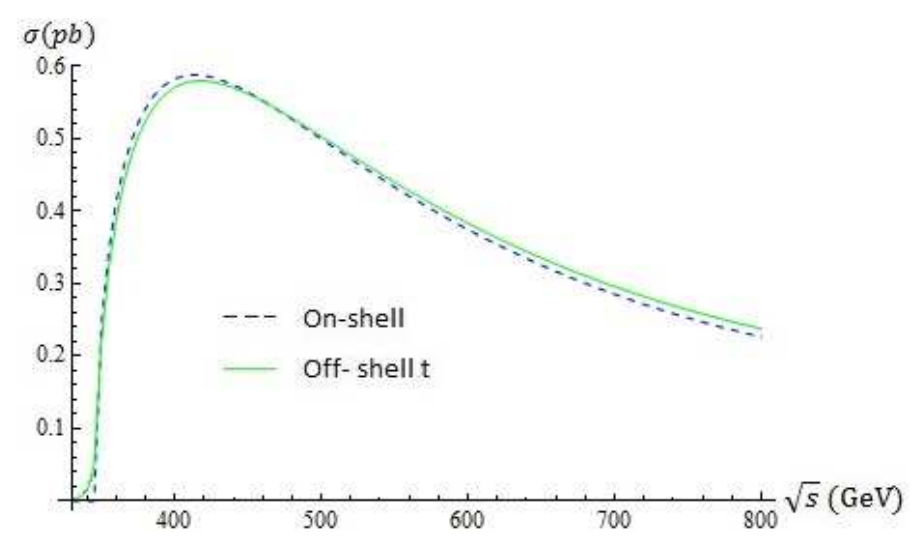

Figure 4: The cross-sections for the process $e^{+} e^{-} \rightarrow t \bar{t}$ and $e^{+} e^{-} \rightarrow b \bar{b} W^{+} W^{-}\left(M_{Z^{\prime}}=3.8 \mathrm{TeV}\right)$

In our calculations we take into account the following corrections:

1. Vertex and self-energy type corrections for stable particles are mainly included into running couplings (2.2).

2. Self-energy corrections for unstable particles are included into function $\rho(m)$, which describes the smearing of UP's masses.

3. Initial state radiation (ISR) is described by spectrum formula $[4,5]$, and the bremstrahlung of $t$-quarks states by vertex $Q$-dependent factor [6].

4. QCD corrections to the top production and decay are described by vertex multiplicative factor [6].

Note, that the corrections are multiplicative and are accounted identically for the total crosssection, angle differential cross-sections and invariant mass distribution. Despite the fact that we added $Z^{\prime}$ boson, influence of the correction isn't changed, see the reference [7].

\section{Comparing with the SM results.}

In this section we show the difference between the MGE model and the SM at considered process. Total cross-sections in the SM and in the model at various $M_{Z^{\prime}}$ are shown in the Fig.5. 


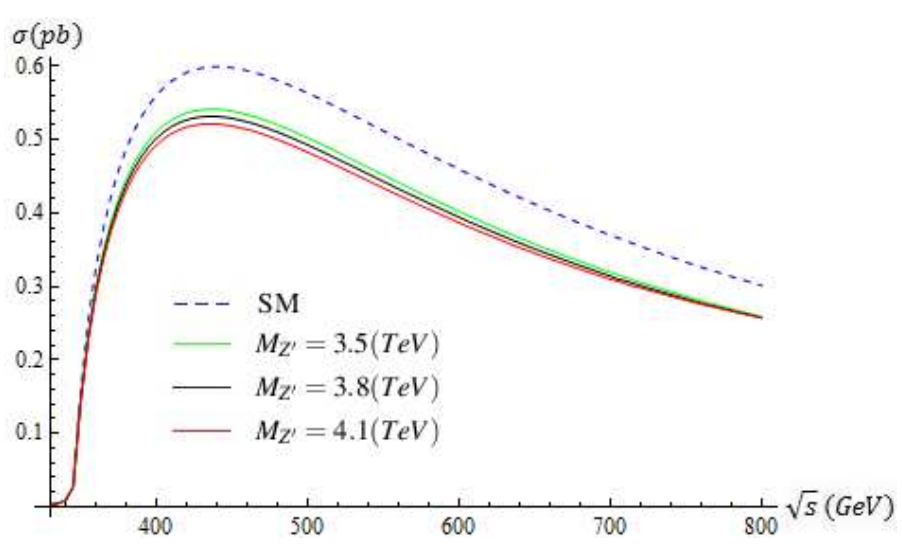

Figure 5: Total cross-section of the process $e^{+} e^{-} \rightarrow b \bar{b} W^{+} W^{-}$

From this picture one can see, that for the model under consideration we have large effect which can easily be registered at the ILC. Note, that we demonstrate the most acceptable by experimental restrictions (2.1) scenario, in other words, at fixed $M_{Z^{\prime}}$ we consider the most acceptable coupling constant $g^{\prime}$ value. Further, in Fig.6, we illustrate angle differential cross-section.

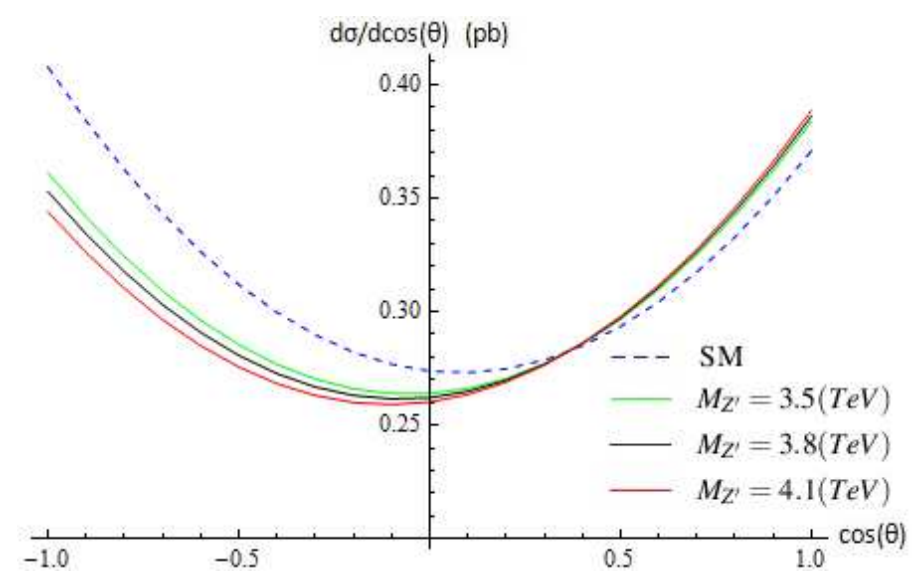

Figure 6: Total cross-section of the process $e^{+} e^{-} \rightarrow b \bar{b} W^{+} W^{-}$

In this case we have principal difference between the SM and MGE of the SM. The asymmetry of the angle differential cross-section is reversed. At last, we demonstrate the invariant mass distribution in Fig.7, where we have a large effect again.

\section{Conclusion}

The production of a $t \bar{t}$ pair and decay in $e^{+} e^{-}$annihilation in the minimal gauge extension of the SM has been analyzed in the frame-work of the SMUP model. This approach significantly simplify calculations and, as shown in [7], the precision of the approach is of the order of 0.1 percent as compared with standard treatment. The method provides a possibility to account for all factorizable corrections. With account of the considered corrections we made a comparison of the 


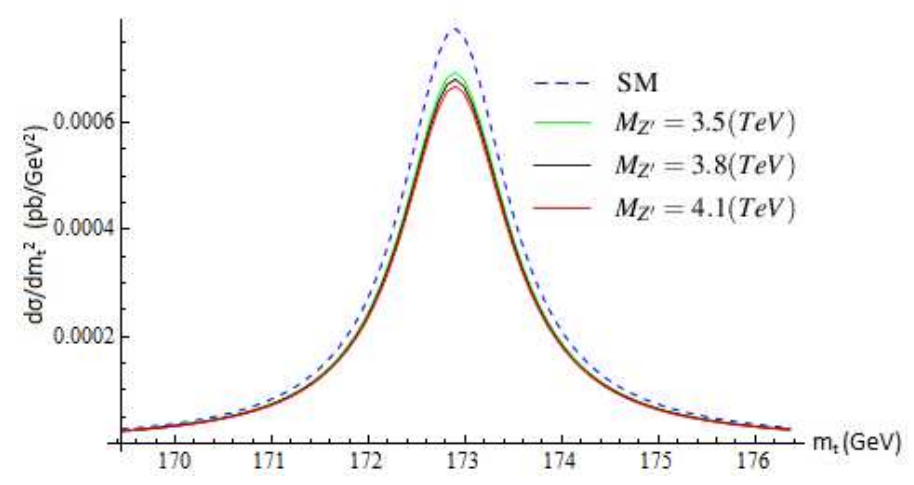

Figure 7: Total cross-section of the process $e^{+} e^{-} \rightarrow b \bar{b} W^{+} W^{-}$

total cross-section, angle differential cross-sections and invariant mass distribution in the SM and MGE of the SM. In spite of $Z^{\prime}$ mass much more than mass of other particles, we had a large effect.

\section{References}

[1] K. Kolodziej et al., Factorizable elektroweak $O(\alpha)$ correction for top quark production and decay at a linear $e^{+} e^{-}$collider, Eur. Phys. C 46, 357 (2009).

[2] V. Kuksa, Finite-width effects in the model of unstable particles with a smeared mass, Int. J. Mod. Phys. A 24, 1185 (2009).

[3] V. Kuksa and N. Volchanskiy, Factorization effects in a model of unstable particles, Int. J. Mod. Phys. A 25, 2049 (2010).

[4] J. Fleischer, W-pair production in $e^{+} e^{-}$annihilation: Radiative corrections including hard nremsstrahlung, Phys. Rev. D 47, 830 (1993).

[5] W. Beenakker et al., in Physics at LEP2, eds. G. Altarelli, T. Sjöstrand and F. Zwirner (CERN 96-01, Geneva, 1996), Vol. 1, p. 79.

[6] A. Denner, Techniques for the calculation of electroweak radiative corrections at the one-loop level and results for W-physics at LEP200, Fortschr. Phys. 41, 307 (1993).

[7] V. Kuksa, D. Vlasenko, The top quark pair production at the LC, in proc. The XXth International Workshop High Energy Physics and Quantum Field Theory, POS (QFTHEP 2011) 033 (2011) 